\title{
MULTI-OPTIMIZATION OF EMPIRICAL MODELS FOR THE MATERIAL EXTRUSION PROCESS
}

\author{
Schuravi N. Mallian ${ }^{1 *}$, Boppana V. Chowdary ${ }^{2 *}$ \\ ${ }^{1,2}$ Faculty of Engineering, The University of the West Indies, Trinidad \\ ${ }^{1}$ Email: schuravi_z@hotmail.com*(Corresponding author) \\ ${ }^{2}$ Email: Boppana.Chowdary@sta.uwi.edu
}

\begin{abstract}
Advances in materials and manufacturing technology and increased competitiveness has led to companies needing to manufacture products more efficiently and rapidly to meet growing market demands. The Additive Manufacturing (AM) process is ideally suited to the fabrication of complex geometries usually impossible with traditional methods furthermore it is capable of fabricating entire assemblies in step without the need for tooling or human involvement.
\end{abstract}

Due to the flexibility and advantages over conventional methods AM has garnered significant attention from the manufacturing sector in meeting market demands. Of the array of available AM processes, Material Extrusion (ME) utilizes a heated thermoplastic filament to construct parts or assemblies via a layer by layer deposition method. This process is not without its own flaws, suffering from accuracy, build time, strength etc., due to the conflicting nature of the process parameters of ME. Therefore, it is critical to understand the shortfalls of ME and classify the factors that directly influence the performance of a part.

This paper focuses on the enhancement of the performance measures of the part in terms of build time, material consumption and max torsional stress. This is accomplished by understanding the influence of the process parameters such as raster width, raster angle, part orientation and layer thickness on the performance measures via statistically valid models and optimization methods. This was accomplished using a Box-Behnken design for the experimental design followed by the multi-objective optimization of the empirical models from which the optimum process settings was determined.

This study has shown that complex a non-linear relationship exists between the process parameters and performance measures. Results show that the Artificial Neural Network had a better fit when compared to the Response Surface Methodology model and it can be a promising alternative for the prediction and optimization of the ME process.

Keywords: Additive Manufacturing, Material Extrusion, Multi-objective optimization https://doi.org/10.47412/WIZL8999

\section{Introduction}

At present the manufacturing industry is under increased pressure due to rising global competition and rapid developments in manufacturing technologies, companies have been put under added pressure to manufacture products and deliver them to fulfil the demands of an ever-evolving market [1][5]. Additive Manufacturing (AM) since its inception has aided companies in accomplishing this goal, with the 
progressive migration from a technique specializing in prototyping to a production capable technology. This has been realized through significant advancements in key areas such as process and materials [2].

Understanding the shortcomings of the AM process is key to its introduction to the industrial and commercial markets as to avoid failures endangering the lives of end users. Also, standardization of parts manufactured by AM techniques needs to be evaluated, as the same part fabricated with different process parameters can display variations in mechanical properties. The reduction of build time and material consumption while maintaining the mechanical integrity is of high importance of most industrial applications. These measures affect the manufacturing cost and functionality of the manufactured part.

Mohamed, Masood, and Bhowmik (2016b) stated that "The material consumption and deposition rate were the major factors in the reduction of build time, and that the deposition rate was dependent upon build parameters such as layer thickness and road width" [3]. It can be said that build time, material consumption and mechanical performance are intrinsically linked to one another. Hence this study looks at finding the optimal build parameters that will improve the max torsional stress while reducing material consumption and build time.

The models generated from this experimentation can assist in comprehending the complex interactions between process parameters and performance measures. Understanding and determining machine parameters based upon the intended end use is key to ensuring proper operational use during its lifetime [4]. Through this, manufacturers can avoid over designing parts and tools thus reducing material wastage and manufacturing time, both of these factors are in turn key cost drivers.

In this paper a systematic methodology is applied in identifying and evaluating key process parameters impacting the performance measures. This methodology employs sampling technique referred to as Response Surface Methodology (RSM) with mathematical modelling and soft-computing methods in solving these multiple objective criterion problems.

The paper is organized as follows: Section 2 briefly discusses the previous research done in the area of Additive Manufacturing; Section 3 presents the experimental process utilized. Section 4 is reserved for the modelling and optimization of the predictive models; in Section 5 the results are briefly discussed. Finally, in Section 6 concludes with recommendations for future work.

\section{Additive Manufacturing}

A critical review of literature spanning a period of two decades has shown that the properties of Additive Manufacturing (AM) parts are intrinsically linked to the various process-related parameters [5], from this review it was seen that the strength of Material Extrusion (ME) parts were dependent upon these five controllable factors; raster angle, air-gap, raster width, part orientation and layer thickness [6].

A study performed by Lee et al. (2007) shows that a compressive specimen has approximately $11.6 \%$ higher strength when the axis of compression is parallel to the build plane, complimentary to this was research from Durgun and Ertan (2014) who reported that build orientation has a greater effect than raster angle on the mechanical strength of ME parts [7][8]. Ziemian, Sharma, and Ziemian (2012) experimentation showed that as the raster angle increased the flexural and impact strength of parts decreased while parts with a raster angle of $0^{\circ}$ exhibited the highest yield strength [9]. Similar to this, Afrose et al. (2016) research showed that samples built $0^{\circ}$ exhibited the highest tensile strength compared to those of $45^{\circ}$ and $90^{\circ}$ [10].

While the reduction of the air gap will lead to more dense components with higher strength fabricated, [11]. This attributed to a reduction in void formation due to low airgap values which increases the bonds between rasters leading to increased structural strength [12]. Raster width was shown to increase the impact and creep resistance of parts, this is a result of thicker rasters reducing the formation of voids in the sub- 
perimeter region directly reducing porosity [3][5]. Thicker rasters have been attributed to the reduction in build time as less time is needed to infill a single interior layer.

The stair stepping effect is a result of layer thickness, generally with a reduction in layer thickness this effect is reduced however at the expense of increased build times. Researchers [5][14][15] have acknowledged the significant impact of layer thickness through experimentation and concluded that the best surface finish and dimensional accuracy was achieved with a minimum layer thickness.

\section{Experimental plan}

Material Extrusion (ME) is one of the Rapid Prototyping (RP) process which can produce functional parts with complex geometries, unlike other RP process however which employ the use of lasers, powers and resins, this utilizes a thermoplastic in the form of filament. Computer controlled nozzles selectively deposit semi-molten filament onto a heated bed, with the nozzles following the contours of the part generated from the $2.5 \mathrm{D}$ sections. After each layer is built the heated bed is lowered by the height of one layer continuing this until the part is completed, bonding of these individual layers is achieved through the process of diffusion welding.

Based on past studies, factors were selected with each respective level considered. These factors are defined as follows:

1. Raster Angle- the direction of the raster relative to the build plate.

2. Raster Width- the width of the rasters used to fill the interior regions of the specimen.

3. Part Orientation- the inclination of the specimen with respect to the $\mathrm{X}, \mathrm{Y}, \mathrm{Z}$ axis.

4. Layer thickness- the thickness of each layer deposited by the nozzle to complete the specimen.

Table 14: Parameter Levels Used for DOE

\begin{tabular}{|l|c|c|c|}
\hline \multicolumn{1}{|c|}{ Build Parameters } & Level 1 & Level 2 & Level 3 \\
\hline Raster Angle (degrees) & 0 & 45 & 90 \\
\hline Raster Width & 0.2 & 0.3 & 0.4 \\
\hline Part Orientation (degrees) & 0 & 5 & 10 \\
\hline Layer Thickness & 0.1 & 0.2 & 0.3 \\
\hline
\end{tabular}

Other process factors are kept at a fixed level in order to maintain a continuity during experimentation. The levels selected for each factor is based upon the permissible minimum and maximum settings recommended by the equipment manufacturer and past experiences. In order to understand the relationship between the process parameters and the performance measures selected, a Design of Experiments (DOE) methodology was employed. Experiments were conducted based on a Response Surface Methodology (RSM) technique, which allows the fitting of second order polynomial models as seen in Eq. (1) which is preferable if curvature is suspected to present in the system.

$$
y=\beta_{0}+\sum_{i=1}^{k} \beta_{i} x_{i}+\sum_{i=1}^{k} \beta_{i i} x_{i}^{2}+\sum_{1 \leq i \leq j}^{k} \beta_{i j} x_{i} x_{j}+\varepsilon
$$

The Box-Behnken from RSM was selected over the Central Composite Design (CCD) as it tends to be less expensive to complete and at no time it produces a run where all factors are set to their extremes, such as all of the highest/lowest settings [16][17]. To develop the Box-Behnken experimental design the number of runs is defined as seen in Eq. (2), with $k$ defined as the factor number and $C_{0}$ the replicate number of 
the central point (Hashemi et al. 2019, 2). The final un-coded DOE design as a result of this is seen in Table 2.

$$
N=2 k(k-1)+C_{0}
$$

Table 2: Box-Behnken Experimental Design

\begin{tabular}{|c|c|c|c|c|}
\hline Experiment & Raster Angle & Raster Width & $\begin{array}{c}\text { Part } \\
\text { Orientation }\end{array}$ & $\begin{array}{c}\text { Layer } \\
\text { Thickness }\end{array}$ \\
\hline 1 & 0 & 0.3 & 5 & 0.3 \\
\hline 2 & 45 & 0.3 & 0 & 0.3 \\
\hline 3 & 45 & 0.4 & 5 & 0.3 \\
\hline 4 & 45 & 0.4 & 5 & 0.1 \\
\hline 5 & 45 & 0.4 & 0 & 0.2 \\
\hline 6 & 90 & 0.3 & 10 & 0.2 \\
\hline 7 & 45 & 0.2 & 10 & 0.2 \\
\hline 8 & 45 & 0.3 & 10 & 0.3 \\
\hline 9 & 45 & 0.4 & 10 & 0.2 \\
\hline 10 & 0 & 0.3 & 5 & 0.1 \\
\hline 11 & 0 & 0.2 & 5 & 0.2 \\
\hline 12 & 90 & 0.2 & 5 & 0.2 \\
\hline 13 & 45 & 0.3 & 10 & 0.1 \\
\hline 14 & 45 & 0.3 & 5 & 0.2 \\
\hline 15 & 45 & 0.3 & 5 & 0.2 \\
\hline 16 & 45 & 0.3 & 0 & 0.1 \\
\hline 17 & 45 & 0.3 & 5 & 0.2 \\
\hline 18 & 0 & 0.3 & 0 & 0.2 \\
\hline 19 & 90 & 0.3 & 5 & 0.3 \\
\hline 20 & 90 & 0.3 & 5 & 0.1 \\
\hline 21 & 45 & 0.2 & 0 & 0.2 \\
\hline 22 & 90 & 0.3 & 0 & 0.2 \\
\hline 23 & 0 & 0.4 & 5 & 0.2 \\
\hline 24 & 45 & 0.2 & 5 & 0.3 \\
\hline 25 & 45 & 0.2 & 5 & 0.1 \\
\hline 26 & 90 & 0.4 & 5 & 0.2 \\
\hline 27 & 0 & 0.3 & 10 & 0.2 \\
\hline
\end{tabular}

Utilizing the experimental design of Table 2 each specimen is fabricated using Raise3D N2 Plus for the respective material consumption, time to manufacture and max torsional stress measurements. The material used for test specimen fabrication is acrylonitrile butadiene styrene (ABS), belonging to the styrene ter-polymer chemical family, it is a hard, tough, heat resistant engineering thermoplastic which is widely incorporated in the automotive sector, housing appliances, luggage and pipe fittings. By dissolving butadiene-styrene copolymer in a mixture of acrylonitrile and styrene monomers, polymerization of the monomers is achieved through the introduction of free-radical initiators [19]. Essentially the three constituents impart a good balance of material properties such as, good impact strength, good heat resistance and rigidity. 
Torsional stress is determined using a SM21 Torsion Testing Machine, the estimated build time and material consumption was taken from ideaMaker 3.3.0 after slicing the part file and compared to the actual recorded values to determine the error percentage.

\section{Modelling and Optimization}

\subsection{Response Surface Methodology}

Analysis of the experimental data obtained from the Box-Behnken design runs is done on Minitab18 software using the full quadratic response model given in Eq. (1). However, before this analysis was done, an initial analysis was done on the data set and it was found that the Max. Stress of experiment 24 was deemed to be an outlier, and the decision was made to remove it, as it skewed the data set when included. The Analysis of Variance (ANOVA) for the second-order regression model is then calculated.

After the initial equations were developed for each model, they were iteratively optimized to allow for the best possible $R_{\text {predicted }}^{2}$ values, this was used as a measure of the models fit because it determines how well the model predicts a response for new observations/inputs. To achieve this the results from each t-test was examined and the P-values of each term was compared to the significance level. An $\alpha=0.05$ was utilized, indicating there is a 5\% risk of concluding that a relationship exists between terms and the response;

- If $\mathrm{P} \leq \alpha$ then the relationship is significant and the user can conclude that there is a statistical significance between the response and the term.

- If $\mathrm{P} \geq \alpha$ then the relationship is not statistically significant, the next step would be to remove one term at a time each time refitting the modelling and examining the P-values and the $R_{\text {predicted }}^{2}$ values. There are instances in the iterative process where removing insignificant terms can reduce the $\mathrm{R}^{2}$ so care should be taken here.

The $R_{\text {predicted }}^{2}$ values after the iterative process for equations (3) - (5) are given as $97.02 \%$ (build time), 96.52\% (material consumption) and $76.88 \%$ (max torsional stress) respectively. The factors for the equations are listed as follows:

$$
\begin{array}{ll}
\text { - } & x_{1} \text { - Raster angle } \\
\text { - } & x_{2} \text {-Raster width } \\
\text { - } & x_{3} \text { - Part orientation } \\
& x_{4} \text { - Layer thickness }
\end{array}
$$

$$
\begin{aligned}
& Y_{1}=560.1-0.221 x_{1}-1157 x_{2}+10.53 x_{3}-2216 x_{4}-0.00176 x_{1}^{2}+981 x_{2}^{2}+ \\
& 2993 x_{4}^{2}+1.056 x_{1} x_{4}-7 x_{2} x_{3}+1650 x_{2} x_{4}-23 x_{3} x_{4} \\
& Y_{2}=10.503-0.00648 x_{1}-7.29 x_{2}+0.3417 x_{3}-12.50 x_{4}+11.46 x_{2}^{2}+11.46 x_{4}^{2}+ \\
& 0.001111 x_{1} x_{3}+25 x_{2} x_{4}
\end{aligned}
$$

$Y_{3}=-13.9+0.573 x_{1}+90 x_{2}+7.43 x_{3}+368 x_{4}-0.00886 x_{1}^{2}+1025 x_{2}^{2}-$

$$
0.0267 x_{1} x_{3}-26.13 x_{2} x_{3}-1774 x_{2} x_{4}+18.31 x_{3} x_{4}
$$




\subsection{Artificial Neural Network (ANN)}

The capability for ANNs to reproduce non-linear functions allows for the possibility of accounting for complex interactions between input and output factors which are not easily modelled (Di Angelo and Di Stefano 2011, 220). The ME process involves a large quantity of factors that interact with one another forming a complex relationship between them, it is often difficult to accurately predict certain output characteristics by conventional methods such as mathematical modelling.

As such a feed-forward back propagation network has been adapted to modelling the ME process. The factors $x_{1}, x_{2}, x_{3}$ and $x_{4}$ are taken as the four input parameters, each of these are represented by one input neuron. Combined with the performance values; build time, material consumption and max torsional stress, they are used to train the model in MATLAB R2018b. Employing a the iterative method in Fig. 1Error! Reference source not found., this process was carried multiple times increasing the complexity of the network until an acceptable network accuracy was achieved.

With the completion of the ANN training, each regression plot is examined to determine if further iterations are necessary, the $\mathrm{R}$ values of the graphs are indicators of the existing relationship between targets and outputs with;

- $\mathrm{R}=1$, there exists an exact linear relationship between targets and outputs

- $\mathrm{R}$ close to 0 , shows that there is no linear relationship existing.

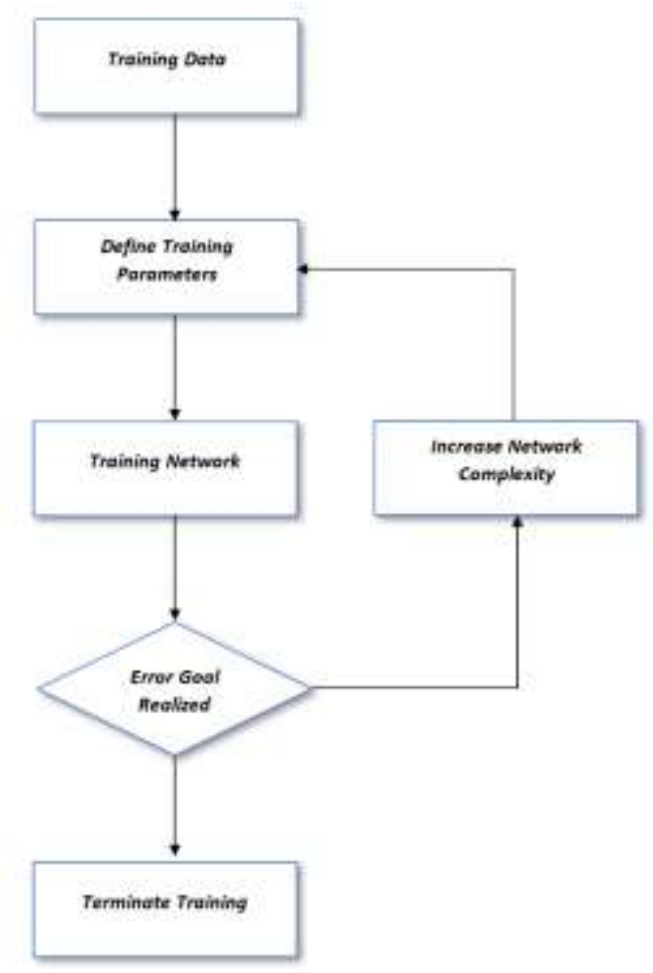

Figure 1: Iterative Process for Developing ANN

The final plot can be seen in Fig. 2, with the three graphs representing the training, testing and overall data. The dashed lines which can be seen in the testing graph presents a perfect result, that is the output of the network maps perfectly on the target data fed into the network at the beginning. While the solid line represents the best fit linear regression line between the outputs and the targets, as seen in the test graph the 
green line is slightly off centre indicating that there isn't an exact linear relationship between the targets and inputs.
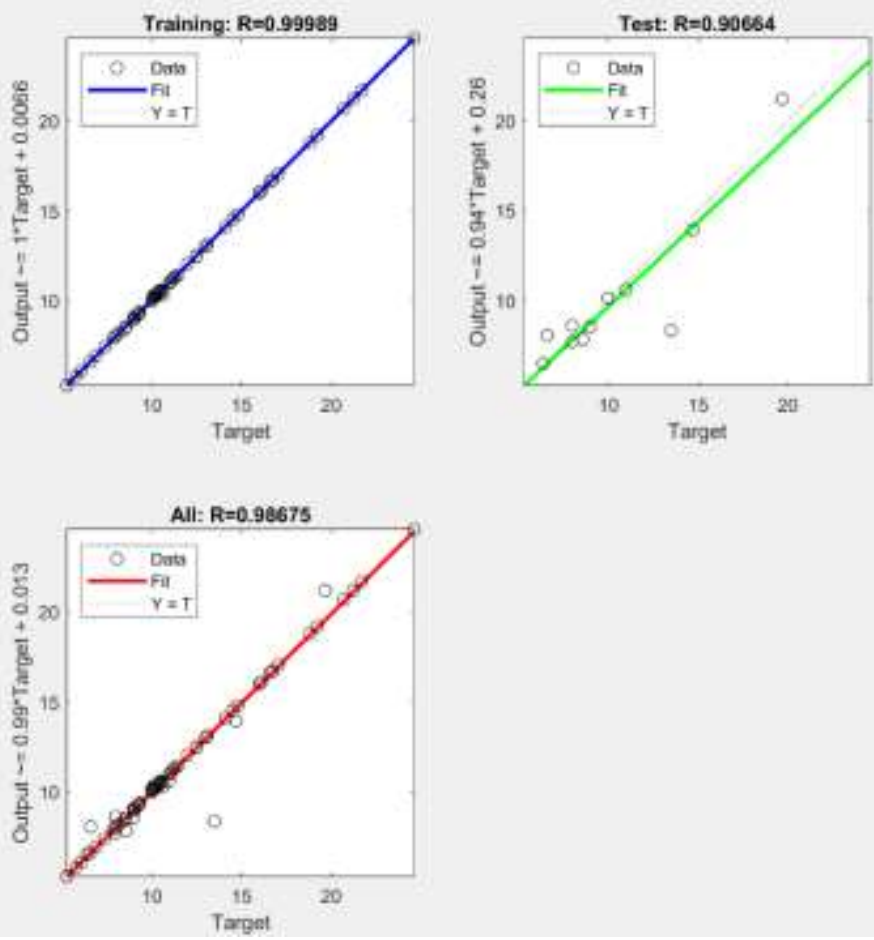

Figure 2: ANN Regression Plot

\subsection{Multi-Objective Optimization}

Each of the models developed via RSM and ANN is subjected to a multi-objective Genetic Algorithm (GA) optimization approach, to obtain the best possible theoretical parameter settings allowing for a desirable set of performance measures. For the implementation of this algorithm a multi-objective minimization problem with four decision variables and three objective functions was formulated as seen below with equation 6 representing the RSM model and equation 7 representing the ANN model both of which are to be minimized.

$$
\begin{gathered}
Y(x)=\left(Y_{1}(x), Y_{2}(x), Y_{3}(x)\right) \\
G(x)=\left(G_{1}(x), G_{2}(x), G_{3}(x)\right)
\end{gathered}
$$

Both objective functions are subjected to identical constraints as seen in Table 1. Table 3 displays the optimum settings from a set of 18 possible optimized solutions, with $f_{1}, f_{2}$ and $f_{3}$ representing build time, material consumption and max torsional stress respectively.

Table 3: Optimum Parameter Settings

\begin{tabular}{|c|c|c|c|c|c|c|c|}
\hline Index & $\mathrm{x}_{1}\left({ }^{\circ}\right)$ & $\mathrm{x}_{2}(\mathrm{~mm})$ & $\mathrm{x}_{3}\left({ }^{\circ}\right)$ & $\mathrm{x}_{4}(\mathrm{~mm})$ & $\mathrm{f}_{1}(\mathrm{~min})$ & $\mathrm{f}_{2}(\mathrm{~g})$ & $\mathrm{f}_{3}(\mathrm{MPa})$ \\
\hline ANN-GA & 62.49554 & 0.398577 & 0.137277 & 0.299908 & 42.71863 & 9.326206 & 64.0815 \\
\hline
\end{tabular}




\begin{tabular}{|c|c|c|c|c|c|c|c|}
\hline RSM-GA & 83.02108 & 0.372367 & 0.010437 & 0.251815 & 43.39755 & 8.767202 & 74.5654 \\
\hline
\end{tabular}

Following the optimization of the models, these values were then used to validate each of them. It should be noted however that due to software (ideaMaker) limitations it is impossible to input the exact process parameter values into the slicing software, Table 4 displays the parameters as they were used in the software and the corresponding performance measures values achieved.

Table 4: Validation Run

\begin{tabular}{|c|c|c|c|c|c|c|c|}
\hline Index & $\mathrm{x}_{1}\left({ }^{\circ}\right)$ & $\mathrm{x}_{2}(\mathrm{~mm})$ & $\mathrm{x}_{3}\left({ }^{\circ}\right)$ & $\mathrm{x}_{4}(\mathrm{~mm})$ & $\mathrm{f}_{1}(\mathrm{~min})$ & $\mathrm{f}_{2}(\mathrm{~g})$ & $\mathrm{f}_{3}(\mathrm{MPa})$ \\
\hline ANN-GA & 62 & 0.40 & 0 & 0.300 & 43 & 9.3 & 150.9207 \\
\hline RSM-GA & 83 & 0.37 & 0 & 0.2518 & 55 & 9.0 & 149.4788 \\
\hline
\end{tabular}

\section{Discussion}

The main objective of this work was to investigate the relationship between the process parameters and the build time, material consumption and max torsional stress. It was seen that part orientation and layer thickness significantly impacted the number of layers of a specimen. High layer thickness combined with low part orientation angle positively impacted the build time and material consumption, as a smaller number of passes were necessary and less material being used to build support for overhang features.

However, with increased orientation angles and decreasing layer thickness the specimens were able to withstand higher torsional stresses before failure, as increased number of layers aided in heat conduction resulting in proper bonding between rasters. Larger raster angles are preferred when there is a need to reduce build time and material consumption as it reduces the number of times the print head needs to change directions, while smaller angles are shown to aid in the strength aspect as it is seen to decrease significantly after $40^{\circ}$.

With an increase in raster thickness there is a decrease in the amount of time spent performing the infill action, however this negatively impacts the material consumption as more material is deposited. While thicker rasters are seen to increase the max. torsional stress, this may be attributed to the fact that thicker rasters tend to retain heat better aiding in the diffusion welding process and also reducing the temperature gradient across the specimen.

Examining the optimized solutions and validation runs data it is seen that the actual values compared to the predicted values of the max. torsional stress values differed greatly. It is possible that with the increased raster angles coupled with high raster widths may have aided in reducing void formation and increased the diffusion welding process reducing the chances of weak bonding between rasters. An increased in layer thickness combined with a part orientation of $0^{\circ}$, reduces the number of heating and cooling cycles leading to non-uniform temperature gradient generating thermal stresses which can lead to improved mechanical strength.

\section{Conclusions and Recommendations}

An attempt has been made in the present work to investigate the effects of four process parameters that is layer thickness, raster width, raster angle and part build orientation on the build time, material consumption and max. torsional stress of ME built specimens. The brittle anisotropic and brittle nature of ME processed ABS was established through the experimental results. Through the use of GA, which is one of the existing evolutionary approaches the optimal parameter settings were found. The investigations have shown the following. 
1. Minimum layer thickness negatively impacts the build time and material consumption especially when paired with a high part orientation. However, specimens were able to withstand higher mechanical loading.

2. Large raster angles should be avoided as it shown to decrease overall strength of specimens, though these large angles are shown to improve the build time and material consumption.

3. Increased raster widths improved the build time and mechanical strength; however, these increases required more material be deposited.

Future work can investigate further performance measures such as creep, vibration damping, cycling fatigue and wear characteristics. Employing the use of another DOE method such as a Q-optimal design as it allows for higher empirical/custom models to be fitter. The quantification of the effects of environmental variables such as build chamber temperature and humidity regarding the performance measures can be explored.

\section{Acknowledgements}

The authors would like to thank for the Campus Research and Publication (CRP) Fund, for granting funds to this project (Ref. CRP.3.MAR18.2).

\section{References}

[1] A. K. Sood, "Study on parametric optimization of fused deposition modelling (FDM) process," 2011.

[2] G. A. Adam and D. Zimmer. On design for additive manufacturing: evaluating geometrical limitations. Rapid Prototyping Journal 21 no. 6, (2015) 662-670.

[3] O. A. Mohamed, S. H. Masood, and J. L. Bhowmik. Mathematical modeling and FDM process parameters optimization using response surface methodology based on Q-optimal design. Applied Mathematical Modelling 40 no. 23-24, (2016) 10052-10073.

[4] C. O. Balderrama-Armendariz, E. MacDonald, D. Espalin, D. Cortes-Saenz, R. Wicker, and A. Maldonado-Macias. Torsion analysis of the anisotropic behavior of FDM technology. The International Journal of Advanced Manufacturing Technology(2018) 1-11.

[5] A. K. Sood, R. K. Ohdar, and S. S. Mahapatra. Parametric appraisal of mechanical property of fused deposition modelling processed parts. Materials \& Design 31 no. 1, (2010) 287-295.

[6] A. K. Sood, R. K. Ohdar, and S. S. Mahapatra. Experimental investigation and empirical modelling of FDM process for compressive strength improvement. Journal of Advanced Research 3 no. 1, (2012) 81-90. [7] C. Lee, S. Kim, H. Kim, and S. Ahn. Measurement of anisotropic compressive strength of rapid prototyping parts. Journal of materials processing technology 187 (2007) 627-630.

[8] I. Durgun and R. Ertan. Experimental investigation of FDM process for improvement of mechanical properties and production cost. Rapid Prototyping Journal 20 no. 3, (2014) 228-235.

[9] C. Ziemian, M. Sharma, and S. Ziemian, "Anisotropic mechanical properties of ABS parts fabricated by fused deposition modelling," in Mechanical engineering: InTech, 2012.

[10] M. F. Afrose, S. Masood, P. Iovenitti, M. Nikzad, and I. Sbarski. Effects of part build orientations on fatigue behaviour of FDM-processed PLA material. Progress in Additive Manufacturing 1 no. 1-2, (2016) 21-28.

[11] S.-H. Ahn, M. Montero, D. Odell, S. Roundy, and P. K. Wright. Anisotropic material properties of fused deposition modeling ABS. Rapid prototyping journal 8 no. 4, (2002) 248-257.

[12] O. A. Mohamed, S. H. Masood, and J. L. Bhowmik. Experimental investigation for dynamic stiffness and dimensional accuracy of FDM manufactured part using IV-Optimal response surface design. Rapid Prototyping Journal 23 no. 4, (2017) 736-749.

[13] O. A. Mohamed, S. H. Masood, and J. L. Bhowmik. Influence of processing parameters on creep and recovery behavior of FDM manufactured part using definitive screening design and ANN. Rapid Prototyping Journal 23 no. 6, (2017) 998-1010. 
[14] J. S. Chohan and R. Singh. Pre and post processing techniques to improve surface characteristics of FDM parts: a state of art review and future applications. Rapid Prototyping Journal 23 no. 3, (2017) 495513.

[15] F. Rayegani and G. C. Onwubolu. Fused deposition modelling (FDM) process parameter prediction and optimization using group method for data handling (GMDH) and differential evolution (DE). The International Journal of Advanced Manufacturing Technology 73 no. 1-4, (2014) 509-519.

[16] M. A. Bezerra, R. E. Santelli, E. P. Oliveira, L. S. Villar, and L. A. Escaleira. Response surface methodology (RSM) as a tool for optimization in analytical chemistry. Talanta 76 no. 5, (2008) 965-977.

[17] D. C. Montgomery, 2017. Design and analysis of experiments. John wiley \& sons.

[18] H. Hashemi, M. Kaykhaii, A. J. Keikha, Z. Sajjadi, and M. Mirmoghaddam. Application of response surface methodology for silver nano-particle stir bar sorptive extraction of heavy metals from drinking water samples: a Box-Behnken design. Analyst(2019).

[19] E. Britannica. (2019, 16/05/2019). Acrylonitrile-butadiene-styrene copolymer. Available:

https://www.britannica.com/science/acrylonitrile-butadiene-styrene-copolymer

[20] L. Di Angelo and P. Di Stefano. A neural network-based build time estimator for layer manufactured objects. The International Journal of Advanced Manufacturing Technology 57 no. 1-4, (2011) 215-224. 